\title{
Proteogenomic biomarkers in colorectal cancers: clinical applications
}

\section{Margherita Binetti , Augusto Lauro , Samuele Vaccari , Maurizio Cervellera \& Valeria Tonini}

To cite this article: Margherita Binetti , Augusto Lauro, Samuele Vaccari, Maurizio Cervellera \& Valeria Tonini (2020) Proteogenomic biomarkers in colorectal cancers: clinical applications, Expert Review of Proteomics, 17:5, 355-363, DOI: 10.1080/14789450.2020.1782202

To link to this article: https://doi.org/10.1080/14789450.2020.1782202 Jun 2020.

Published online: 22 Jun 2020.

Submit your article to this journal $₫$

Џ Article views: 29

Q View related articles $₫$

View Crossmark data $₫$ 
REVIEW

\title{
Proteogenomic biomarkers in colorectal cancers: clinical applications
}

\author{
Margherita Binetti ${ }^{a}$, Augusto Lauro ${ }^{a}$, Samuele Vaccari ${ }^{b}$, Maurizio Cervellera ${ }^{a}$ and Valeria Tonini ${ }^{a}$

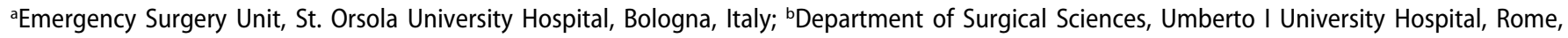 \\ Italy
}

\section{ABSTRACT}

Introduction: Colorectal cancer (CRC) is one of the leading cancers in terms of incidence and mortality, rate requiring a multidisciplinary approach. The discovery of specific CRC biomarkers has caused a paradigm shift in its clinical management.

Areas covered: The aim is to illustrate the possible clinical applications of CRC biomarkers through an updated literature review (from 2015 to 2020) based on the PubMed database. A relationship between cancer localization and genetic profile has been identified. Nowadays, the tumor markers are largely used to select patients that could really benefit from a specific type of adjuvant therapy, in order to optimize treatment programs, especially in metastatic patients. This review highlights both CRC biomarkers' advantages and critical issues.

Expert opinion: New biomarker discoveries allow to set noninvasive tests that could increase patient's compliance with therapy. They also permit a cost-effective early diagnosis, as well as patienttailored treatments, improving the overall survival. The CRC biomarkers could also have a prognostic value, and usually, they are included in follow-up programs. However, despite the continuous progression of new technologies, their clinical validation is still debated. In this context, additional clinical studies are still necessary to identify, among potential markers, the most effective ones.
ARTICLE HISTORY

Integra15 June

2020Received 10 May 2020

Accepted 9 June

2020Received 10 May 2020

Accepted 9 June 2020

\section{KEYWORDS}

Biomarkers; colorectal cancer; early diagnosis; screening; tailored therapy

\section{Introduction}

Colorectal cancer (CRC) is internationally recognized as one of the leading malignant cancers in terms of incidence and mortality rate [1]. At diagnosis, $25 \%$ of CRC patients already have liver metastases, while $50 \%$ develop them during the disease course [2]. The clinical approach shows some critical issues, especially related to screening, diagnosis, personalized therapy, efficacy of follow-up, and prognosis. This is why its management requires multidisciplinary tasks [2] and gastroenterologists, radiologists, surgeons, and oncologists are often involved altogether.

3,4 to obtain an early diagnosis are only two of the key elements driving to biomarker research [5]. Historically, CRC therapeutic approach relied on surgery [6], but the discovery of oncological CRC markers has revolutionized the therapeutic algorithms [7]. Few decades ago, metastatic patients were considered incurable [8], but more recently several tumor antigens, useful for tailored immunotherapy, have been identified [9]. Although prognosis depends on many factors, tumor genetic and epigenetic profile is largely the most considered one [3], and, because of all clinical implications, there is increasing international focus on their mechanism of alterations [10].

It has been shown that CRC is a heterogeneous disease [11]. Already in 2000, Hanahan and Weinberg [12] highlighted the concept of 'heterogeneity.' According to the authors, the cancer's complexity is not composed of homogeneous cells, but by many distinct clonal subpopulations, demarcated by different features in terms of differentiation, proliferation, and vascularity. Part of genetic diversity could be reflected in histopathological different subtypes. This represents the starting point for the subsequent development of biomarkers' research [12].

These molecular differences among CRCs could explain both the variability in treatment's response and the necessity of new predictive biomarkers [8]. Many molecular targets have been identified, but due to few important limitations, their clinical application is not always possible [3]. The most important limitation is represented by the poor availability of international, multicentre studies, also due to the high cost of analysis. For example, for CTCs' detection the CellSearch system is the only approved method by the US Food and Drug Administration [3].

However, new frontiers of research are under investigation, such as microRNAs (miRNA) [13]. Especially for metastatic CRC patients (mCRC), molecular biomarkers could represent a fundamental change in the basic concepts, experimental practices, and a paradigm shift in therapeutic management [14].

\section{Colorectal cancer (CRC)}

Internationally the incidence and mortality rate of $C R C$ is differently reported and it depends on HDI (Human Development Index) [1], a summary measure of average achievement in key dimensions of human development such as a long and healthy life, being knowledgeable and having a decent standard of living. Recently, in countries with

CONTACT Augusto Lauro augustola@yahoo.com Emergency Surgery Unit, Pavillion 5, St Orsola University Hospital, Bologna 40138, Italy This article has been republished with minor changes. These changes do not impact the academic content of the article

(c) 2020 Informa UK Limited, trading as Taylor \& Francis Group 
Article highlights

- $\mathrm{CRC}$ is one of the leading cancers in terms of incidence and mortality rate.

- Biomarkers could have diagnostic, predictive, and prognostic roles.

- The use of CRC biomarkers could be involved in screening such as non-invasive tests for early diagnosis.

- Some genetic and molecular differences have been observed between the right-sided and left-sided CRCs.

- Proteomics in CRC can be used to identify the tailored appropriate therapy, especially in metastatic CRC patients.

- Other clinical studies are necessary to validate a panel of biomarkers that could be internationally used in clinical practice.

medium-high $\mathrm{HDI}$, both the $\mathrm{CRC}$ incidence and mortality rates have increased because of national screening programs, while in countries with very high $\mathrm{HDI}$ an increase of incidence and a decrease of mortality have been observed. Finally, the incidence and mortality rates have been both decreased in the top, highest HDI countries [1].

CRC can be sporadic, with slower development through the adenoma-carcinoma sequence, or hereditary [15]. In hereditary $\mathrm{CRC}$, it is possible to make a difference between proximal and distal localization also based on pathological features [16]. Proximal cancers are often less aggressive. They have a diploid DNA, microsatellite instability, and mutations in mismatch repair (MMR). In contrast, distal one's behavior is generally more aggressive, presenting with aneuploid DNA, mutations in adenomatous polyposis coli (APC), KRAS, and p53 genes [16].

Clinical presentation between right-sided and left-sided CRC has been known as different for a long time [17] (Figure 1). But nowadays it is clear that these differences are much more extended and they also involve pathological characteristics, molecular biomarkers, and prognosis as well [18].

Screening programs represent the main tools to obtain early cancer detection [19]. In this context, the greater advantage of colonoscopy over other methods depends on its possibility to be often diagnostic and therapeutic at the same time [20]. However, a 2-step screening program, consisting of predictive biomarkers' analysis followed by colonoscopy has been considered [21].

The American Joint Committee on Cancer (AJCC) has provided a new complete CRC staging system [22] (Table 1). Compared to the previous ones, the last edition (AJCC 8th edition, 2018) focuses more on molecular markers' roles and has highlighted the importance of personalized approach for diagnosis, treatment, and prognosis [22]. The CRC therapeutic algorithm takes into consideration different features related to both cancer's and patient's presentation [23]. Recent pathophysiological knowledge has increased the set of medical and surgical therapeutic options [24]. The surgical approach represents the basis of curative treatment for both right- and left-sided colonic cancers and a correct surgical technique provides optimal results [25].

Differently from the past, nowadays a surgical role is also considered possible in metastatic CRC (mCRC), especially for synchronous liver metastases [26]. However, for patients with advanced CRC, the major interest is for new therapies and prognostic perspectives which are based on biological neoplastic

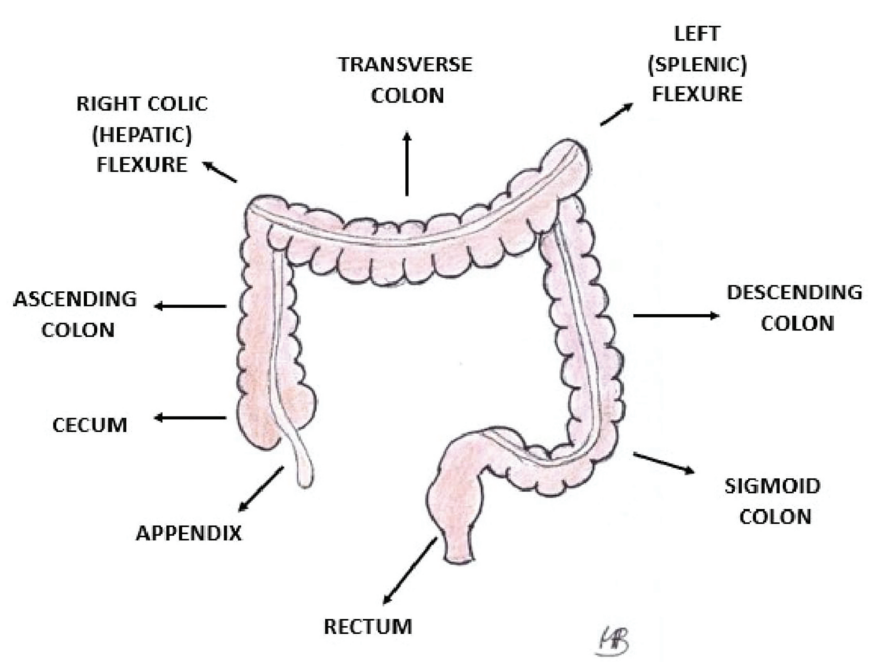

Figure 1. Different regions of the colon.

features $[5,13]$. Recently, the utility of 'tailored therapy' has been proven in many studies, based on the fundamental paradigm that patients respond to biological therapies in different ways $[27,28]$. The term 'tailored therapy' is used to indicate a treatment based on patients' individual characteristics and specific cancer's histological pattern [8]. It allows to have more personalized therapeutic programs and it emphasizes the importance of the future researches. The biomarker-driven approach can strongly optimize their outcome [29].

\section{Proteogenomic biomarkers}

A molecular biomarker is defined by the National Cancer Institute $(\mathrm{NCl})$ as the biological molecule found in blood or other body fluids or tissues, that is a sign of a normal or abnormal process or of a condition or disease' [5].

The continuous research of new CRC biomarkers represents the effort to develop noninvasive tests in order to be used in the algorithm of the disease management [30]. In this context, blood proteins represent an ideal source, but biological markers could also be detected in other patients' tissues. The cellfree circulating DNA (cfDNA) and the circulating tumor cells (CTCS), for example, can be studied using the liquid biopsy technique, and they both present diagnostic and prognostic clinical roles [3]. Among the noninvasive exams, stool tests should also be mentioned, and the detection of SEPT9, NDRG4, and SDC2 DNA-methylation has been proposed [31]. The use of stool samples for early detection is based on the principle that neoplastic cells are continuously eliminated in the intestinal lumen, mixing with stool [31].

On the other hand, some examples of invasive tissue biomarkers are cyclin E, p27kip 1, ki67, ZNF33, and some miRNAs (for example mir-21, mir-25, mir-31, mir-124) [3].

The knowledge of genetic, proteomic, and epigenetic features is due to new technologies that allow to set a molecular map of CRC [32]. In order to clarify CRC biomarkers' clinical roles, it is useful to classify them according to their own biomolecular features (Figure 2). 
Table 1. TNM staging of colorectal carcinoma (AJCC edition) [22].

\begin{tabular}{|c|c|c|c|}
\hline & $\mathrm{T}$ & $\mathrm{N}$ & M \\
\hline STAGE 0 & Tis & No & MO \\
\hline STAGE IA & $\mathrm{T} 1$ - T2 & No & Mo \\
\hline STAGE IIA & T3 & NO & Mo \\
\hline STAGE IIB & T4a & No & Mo \\
\hline STAGE IIC & T4b & No & Mo \\
\hline STAGE IIIA & $\begin{array}{c}\mathrm{T} 1 \text { - T2 } \\
\mathrm{T} 1\end{array}$ & $\begin{array}{c}\text { N1/N1c } \\
\text { N2a }\end{array}$ & $\begin{array}{l}\text { MO } \\
\text { Mo }\end{array}$ \\
\hline STAGE IIIB & $\begin{array}{l}\text { T3 - T4a } \\
\text { T2 - T3 } \\
\text { T1 - T2 }\end{array}$ & $\begin{array}{c}\text { N1/N1c } \\
\text { N2a } \\
\text { N2b }\end{array}$ & $\begin{array}{l}\text { MO } \\
\text { MO } \\
\text { MO }\end{array}$ \\
\hline STAGE IIIC & $\begin{array}{l}\text { T4a } \\
\text { T3 - T4a } \\
\text { T4b }\end{array}$ & $\begin{array}{c}\text { N2a } \\
\text { N2b } \\
\text { N1 - N2 }\end{array}$ & $\begin{array}{l}\text { MO } \\
\text { MO } \\
\text { MO }\end{array}$ \\
\hline STAGE IVA & anyT & anyN & M1a \\
\hline STAGE IVB & anyT & anyN & M1b \\
\hline STAGE IVC & anyT & anyN & M1c \\
\hline
\end{tabular}

$\mathrm{T}=$ tumor; $\mathrm{N}=$ lymph nodes; $\mathrm{M}=$ metastasis.

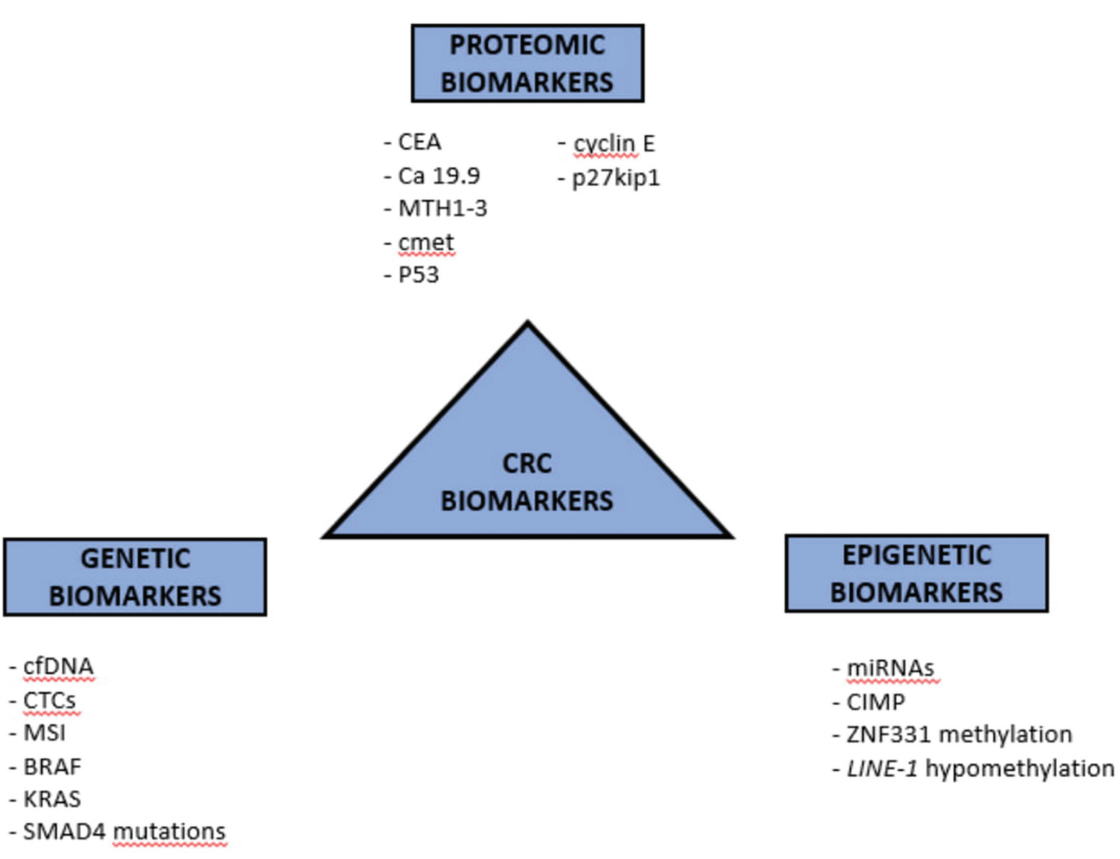

Figure 2. Genetic, proteomic and epigenetic CRC biomarkers.

\subsection{Genetic biomarkers}

A first group of CRC tumor markers is formed by 'genetic biomarkers,' and among them, the presence of short tandem repeat DNA sequences, also known as microsatellite instability (MSI), is relevant [33]. Some other prognostic markers like BRAF and KRAS belong to the same group [3]. A relevant genetic change in CRC is the SMAD4 mutation, which is followed by 
the loss of SMAD4 protein expression [34]. SMAD4 gene mutations have been observed in 2-20\% of CRC patients [3].

The BRAF is part of MAPK signaling pathway, while the KRAS is characterized by GTPase intrinsic activity [35]. However, these last two markers are both parts of the epidermal growth factor (EGFR) pathway, which is recognized as a major driver in CRC development [8].

\subsection{Proteomic biomarkers}

A second group consisting of 'proteomic biomarkers.' In the past, the attention was focused on gene mutation's status, but nowadays the proteome detection and its modifications have become crucial [5]. Three steps follow each other in proteomic biomarkers' research. The first one is the discovery, in which proteins can be extracted and studied using mass spectrometry, while the second one is the verification. It consists of quantification of the target proteins using different techniques, such as SRM-MS (selected reaction monitoring-mass spectrometry), SWATH (sequential window acquisition of all theoretical mass spectra), or MRM-MS (multiple reaction monitoring-mass spectrometry). The last one is the validation [5].

This large group involves the Carcinoembryonic Antigen (CEA) [3]. Both the tissue carcinoembryonic antigen (t-CEA) and the serum carcinoembryonic antigen (s-CEA) are primarily used for CRC diagnosis and management, but their validity is still controversial [36]. A possible Carbohydrate Antigen (Ca19.9) role in post-surgery surveillance has been considered, but proofs related to this topic are poor in literature [37]. Some other proteomic biomarkers are the MTH1-3 [38] and the Mesenchymal-epithelial transition (cmet) [39]. In addition, the P53 mutation pattern is also involved in CRC pathogenesis [40].

\subsection{Epigenetic biomarkers}

A quite new group is formed by 'epigenetic biomarkers,' and among them, miRNAs and CPG island methylator phenotype (CIMP) have a primary role [3]. The epigenetic aberrant methylation of ZNF331 is presented as an independent prognostic factor, with a sensitivity and specificity rate of $71 \%$ and $98 \%$, respectively [41]. In reverse, the Long interspersed nucleotide line 1 (LINE-1) epigenetic hypomethylation can be related to advanced cancer's presentation and it negatively impacts patients' survival [42].

\section{CRC biomarkers in clinical practice}

Biomarkers play a fundamental role in many steps of CRC disease management, especially in diagnosis and treatment [43]. They can be summarized as 'diagnostic biomarkers,' used for early diagnosis and risk stratification, 'predictive biomarkers,' used to predict patients' response to specific treatments and finally 'prognostic biomarkers,' used to predict CRC natural history [10]. They should all demonstrate some fundamental criteria to be currently used in clinical practice, such as high sensitivity and specificity rates. Moreover, they should also be easily reproducible among laboratories [44].
In order to match CRC biological features to clinical ones, an international classification has been recently approved [45]. The 'Consensus Molecular Subtypes' (CMS) report both clinical and biological cancer's characteristics, and in this overview, four categories have been identified, from CMS1 to CMS4 [46].

The CMS1 (14\%) involves MSI (microsatellite instability) tumors with strong immune activation, while the chromosomal instability (CIN), typical WNT, and MYC pathways are part of CMS2, which is formed by epithelial cancers (37\%). It represents the most frequent group. The CMS3 (13\%) is an epithelial one as well and it is characterized by metabolic dysregulation pathways. Finally, the CMS4, consisting of mesenchymal tumors (23\%), presents the epithelial-mesenchymal transition (EMT) pattern with a TGF- $\beta$ activation. Because of this reason, the CMS4 has the poorest prognosis [45].

In order to verify if genetic differences of CMS 1-4 groups correspond or not to the protein level, many FluorescenceActivated Cell Sorting (FACS) analyses were performed [46]. A distinction between CMS1/CMS4 and CMS2/CMS3 was obtained. For example, CD166/CD44 expression was undetectable in groups 1 and 4, while CXCR4 was highly expressed in group 4 [46]. The detection of MSI can be performed using MLH1 and MSH2 proteins. Only samples with a high level of these proteins could be considered part of CMS1 [45]. It is clear, according to the authors, that there is not a perfect correspondence between genetic and protein level. Therefore, the genetic and protein levels should be kept distinct $[45,46]$. The translation from medical literature to current clinical use represents a real challenge, and only a small number of studied biomarkers have been finally validated [47].

We report a clinical overview of CRC biomarkers:

\subsection{Hereditary pre-cancerous conditions}

In this setting, biological biomarkers could be used to differentiate between sporadic and hereditary cancer [5]. Among hereditary conditions, different elements have been described, such as the Familial Adenomatous Polyposis (FAP), and the Hereditary Non-Polyposis Colon Cancer (HNPCC), also known as Lynch Syndrome [48]. The FAP is an autosomal disorder, involving the APC gene, part of Wnt/beta-catenin pathway [49], while the Lynch Syndrome is the most frequent syndromic disease associated with $\mathrm{CRC}$, representing $3 \%$ of new diagnoses [50]. It is a genetic condition due to DNA mismatch repair (MMR), expressed by few gene mutations responsible for error accumulation in DNA. In specific, the MLH1 gene (50\%), MSH2 (40\%), MSH6 genes (7\%-10\%), and PMS2 gene (5\%) are often involved [5]. Finally, the PJS is also related to an increased cancer risk, which has been related to P53 pathway. The P53 activity test could be useful in the clinical management of the disease [51]. There are also some other inherited syndromes, such as MUTYH-associated polyposis and Cowden/ PTEN hamartoma syndrome [52].

\subsection{Screening and diagnosis}

In the last decades, several different noninvasive stool-based screening tests have been proposed, such as the guaniac-Fecal Occult Blood Test (gFOBT), the Fecal Immunochemical Test 
(FIT) and the newer fecal DNA (Multitarget stool DNA, MTsDNA) $[4,53]$. The first one detects the presence of blood in feces, taking advantage of the peroxidase activity of heme, while the second one is an antibody to human globin [4]. All these noninvasive exams could represent a primary screening test; however, colonoscopy is now considered the gold standard because of its higher sensitivity and specificity. It has both diagnostic and therapeutic features [53]. The procedure is performed by a probe and it allows to detect precancerous intestinal polyps [53]. In order to have a correct and complete view of intestinal walls, particularly important is preendoscopic preparation of the bowel while sedation is also required during the procedure [4]. Even though it is quite unusual, this invasive procedure can be complicated by perforations, Gl bleedings, cardiopulmonary events, and relevant abdominal pain [54]. In this context, there is a big effort to discover a noninvasive procedure, such as diagnostic biomarkers [55]. The CEA and the CA 19-9, currently used in many other malignancies [56], present a poor role in CRC. This issue has soon stimulated the search of new biomarkers' panels. For example, the SDC2, SFRP2 methylation, and KRAS mutations have shown a sensitivity rate of $91.4 \%$ in CRC screening [55]. Some authors suggest the necessity of multiple biomarker use to detect CRC [57]. According to Gao Y. et al. [57], CA19-9, CA74-4, and CA125 are related to poor differentiation of CRC, while the CEA, CA19-9, CA74-4, and CA125 are also related to the presence of lymph-nodes and distant metastases. The performance of the FDA-approved screening test named Cologuard (stool DNA-based screening test) was not satisfactory too [55].

However, to date there is still no biomarker able to replace colonoscopy because there is no international consensus about a specific one and the endoscopic procedure has both a diagnostic and a therapeutic role. Self-evidently biological markers, which are less invasive, cannot allow removal of intestinal polyps [53]. Nevertheless, a possible testing of aberrant genetic methylation in feces has been also tried and some authors concluded that SEPT9, NDRG4, and SDC2 methylation in stool could be detected as a potential biomarker for early screening [31].

\subsection{Right-sided and left-sided localization}

The biological, pathological, clinical, and prognostic differences between proximal (right-sided) and distal (left-sided) CRC localization have been clear for a long time [58]. But quite recently, due to the development of new research techniques, some genetic and molecular differences between the two groups have emerged [17]. The P53 mutations with microsatellite stability pathway (MSS) have been observed as more associated with left-sided cancer, while MSS phenotype with KRAS mutations has been reported more frequently in right-sided malignant neoplasia [59]. A molecular marker of deficient mismatch repair (dMMR) characterizes the proximal colonic cancer, leading to a higher risk related to the increased number of infiltrating lymphocytes [8]. Finally, the reduced CDX2 expression has been described in right-sided colonic cancers as well [3].

\subsection{Therapeutic approaches}

The surgical approach can be guided by biological biomarkers [43]. In fact, it could be advisable to determinate CEA expression before surgery, especially for metastatic patients, because likely CEA can determinate life expectancy, potentially influencing the surgeon's clinical choices [43]. For early detected cancer (stage I), the surgical approach should not be followed by adjuvant therapy because less than $10 \%$ of the patients have a disease recurrence [8]. Localized cancer's diagnosis is related to a 5-year survival rate up to $90 \%$ [5].

However, the introduction of noninvasive tests has caused a greater paradigm-shift in oncological therapy rather than in the surgical approach [5]. One of the most important roles of biological biomarkers is now played in adjuvant therapy [8]. Even though the oncological decision-making process is primarily based on CRC staging, the daily practice has highlighted the need for predictive and prognostic biomarkers because of singular patient variability [8]. Biomarkers offer oncologists the opportunity to identify which patients could really benefit from a specific adjuvant therapy, in order to optimize the therapeutic program [10]. Predictive biomarkers are able to indicate both the sensitivity and the resistance to the treatment of particular types of cancer [8].

Nowadays, testing KRAS, NRAS, and MSI has become a fundamental disease-management step to set a therapeutic program requiring personalized therapy, especially for metastatic patients [60]. Studying KRAS and NRAS mutations is the key to select patients that could respond to Cetuximab and Panitumumab anti-EGFR program therapies [61]. Over the last ten years the EFGR pathway has become the first target therapy and $25-40 \%$ of CRC patients are quadruple-negative (KRAS/ NRAS/BRAF/PI3KCA wild type) [62]. This condition is related to a low possibility to have a good response to all target therapies [62]. In refractory cases, a study of Her-2 amplification and NTRK1, ALK, or ROS1 targets could also be considered [61].

In mCRC patients treated with Bevacizumab, a possible prognostic role of hERG1 and aHIF-2a has been highlighted. They represent proteins related to angiogenesis pathway [63].

In this framework, because of their stability in blood, miRNAs have achieved a primary role to predict treatment response of $\mathrm{mCRC}$ patients [14]. Many studies have reported miRNA clinical roles, especially for patients treated with 5-fluorouracil (5-FU) [64,65], oxaliplatin [66-68], and FOLFOX [69]. However, all these researches are usually completed 'in vitro' only, with lack of 'in vivo' evidences [14]. For dMMR pattern, some previous studies have indicated a resistance to 5-FU but a sensitivity to Oxaliplatin [70].

\subsection{Follow-up}

According to the 'American Society of Colon and Rectal Surgeons' and the 'American Joint Committee on Cancer,' the CRC postsurgical follow-up must involve physical examinations, CEA testing, and imaging surveillance [71]. Therefore, CEA is currently used to monitor CRC disease history, but this marker has both low sensibility and sensitivity rates [72]. In addition, it remains still unclear whether it could predict cancer recurrence. However, it seemed to be an independent prognostic factor after surgery for 
stage I-III patients [73]. Some encouraging results have been obtained in mCRC follow-up. In fact, tumor-specific mutation and NPY methylation, both performed on circulating DNA, seemed to be positively associated with imaging findings [72].

\subsection{Prognosis}

A prognostic biomarker provides information about cancer natural history and its potential outcome, independently of treatments [8]. According to Duan's point of view [3], there is still a great effort to identify new valid prognostic biomarkers. For example, the cfDNA expression has been used for tracking CRC disease relapse [74]. The CTCs have been related to poor prognosis [75], while MSI-H could predict better survival [3]. The MSI high expression has revealed a better overall survival for stage II patients [76]. The stage II patients, together with the stage III ones, represent $15 \%$ of the total amount [76].

However, the KRAS and BRAF pathways' role in CRC prognosis is still controversial. According to few studies, the mutation of these biomarkers could influence the overall survival [77]. On the other hand, in few recent studies, the effective correspondence between KRAS-BRAF mutations and poor prognosis has been excluded [78]. The SMAD4 loss or mutation may result in cancer progression [79].

In this framework, epigenetic biomarkers such as CIMP, ZNF331, and LINE1 biomarkers [3] can also be involved. For example, the poor prognostic prediction of CIMP could be specifically observed only in patients with MSS and MSS BRAF mutated cancer [80].

\section{Conclusions}

Recently, CRC management has changed from a 'general disease' approach to a 'single patient' tailored one. In this paradigm shift, proteogenomic biomarkers have played a primary role. Their usefulness has been hypothesized for screening, diagnosis, localization, therapy, and prognosis. An overview of their clinical applications has been reported in Figure 3. In this context, they present some clear clinical advantages such as the chance to be used as noninvasive tests, increasing patient compliance both in early screening and follow-up. In addition, biomarkers provide a description of cancer features, which could implement a patient-tailored therapeutic approach. However, taking into consideration a large database of potential biomarkers that have been studied so far, their clinical validation is still debated.

Therefore, the continuous research of innovative technologies to investigate new biomarkers should be followed by clinical studies. The possibility to set new international protocols of CRC management, involving validated biomarkers, is the ultimate goal and all efforts should be headed in this direction.

\section{Expert opinion}

Nowadays many aspects of CRC proteogenomic are internationally investigated. Our literature review is focused on the clinical application of CRC biomarkers. It has been completed by checking the Pubmed database. All the mentioned publications are part of the most recent CRC literature (from 2015 to 2020). Some older articles have been included in this report, because of their impact on subsequent studies. For the CRC staging, the latest AJCC 8th edition has been used.

Serum proteomics increased the possibility to discover novel markers, providing that they would reflect the early stage of cancer and behave as a prognostic prediction of CRC.

Biomarkers should play an important role in the detection and treatment of patients with CRC, but as previously remarked CEA and CA 19-9 (currently used in many other

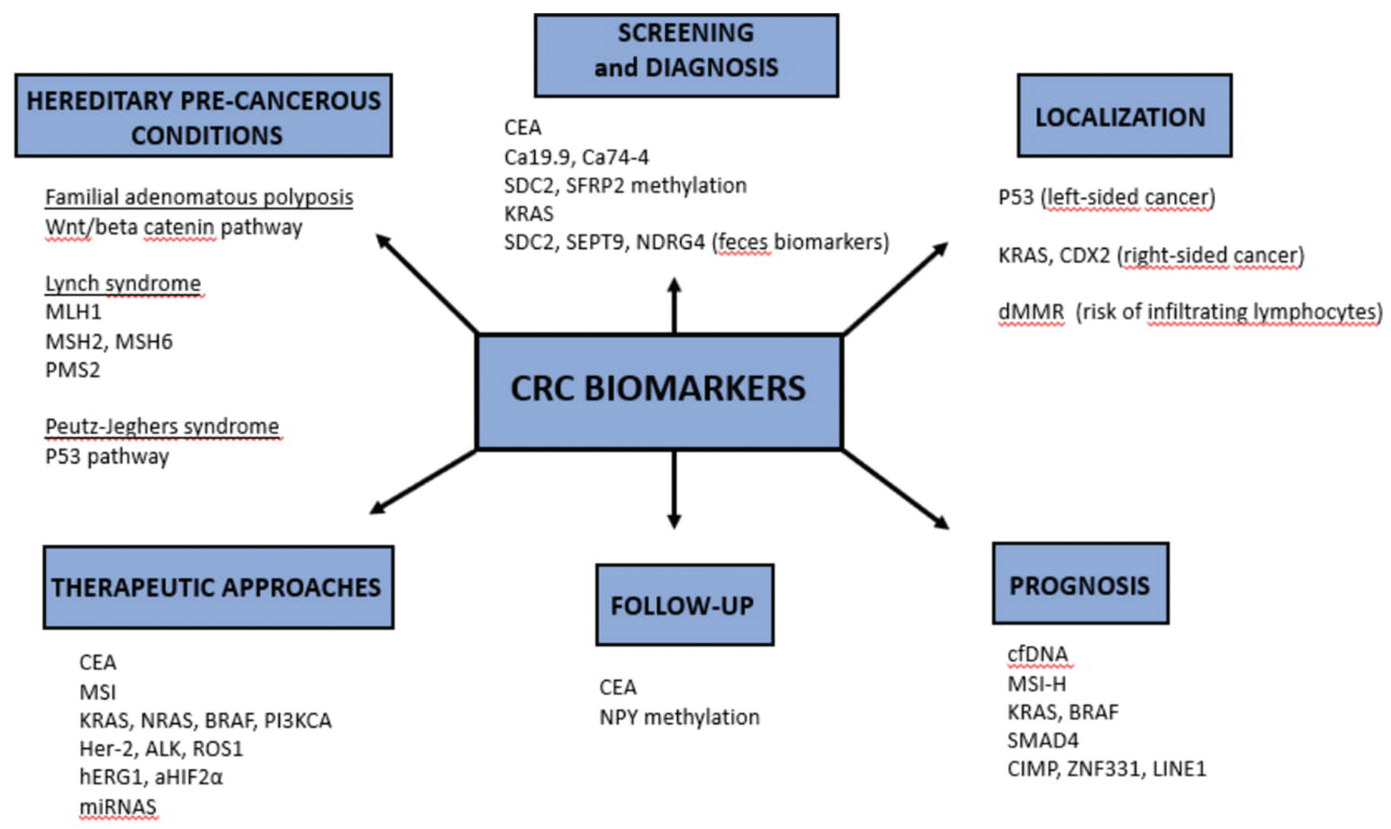

Figure 3. Overview of CRC biomarkers and their clinical applications.

The diagram sums up the usefulness of potential biomarkers in the most important steps of CRC management: hereditary condition exclusion, screening and diagnosis, cancer localization, therapeutic options, follow-up, and prognosis. 
malignancies) present a poor role in CRC, due to low sensitivity especially in early stages.

Ten years ago [81], a meta-analysis provided a list of CRCassociated tissue proteins discovered in multiple studies, employing tandem mass spectrometry. The authors stated that up to year 2010, only a limited number of CRCassociated proteins were validated in serum for noninvasive testing in CRC. The situation has changed ten years later, at least from the laboratory point of view: the screening has been implemented by finding novel biomarkers able to recognize either the predisposition or the early stage of the disease, potentially improving the survival rate. In a recent metaanalysis of the literature [82], MST1/STK4 (Mammalian STE20like protein kinase 1/Serine threonine kinase 4) showed a sensitivity of $68 \%$ and a specificity of $78 \%$; S100A9 (S100 calcium-binding protein A9) achieved a sensitivity of $72 \%$ and a specificity of $83 \%$; and TIMP1 (Tissue inhibitor of metalloproteinases 1) obtained a sensitivity of $42 \%$ and a specificity of 88\%. MST1/STK4, S100A9, and TIMP1 showed diagnostic efficiency and excellent performance for CRC detection by means of a simple blood sample, but their widespread clinical application is still far away.

Biomarkers should also have the possibility to change treatment algorithms by selecting the proper chemotherapeutic drugs across a broad spectrum of patients. As previously remarked, there are attempts to personalize chemotherapy based on the presence or absence of specific biomarkers, and the goal of future research in proteomics will be to identify those markers that could allow a noninvasive and cost-effective diagnosis, as well as to recognize the best prognostic panel and define their predictive power for available treatments.

Proteogenomic studies represent the new frontier of proteomics applied to CRC. The proteogenomic characterization plus integrative and comparative genomic analysis provides a new paradigm for understanding human malignant colonic and rectal liver metastases.

A 2018 study [83] revealed that two sites (DMRTB1R202 H and PARP4V458I) frequently mutated only in a liver metastatic cohort of patients and displayed dysregulated protein abundance. Another example is given by the improvement of patients' stratification due to the selection of CRC patients affected by liver metastases, eligible for anti-EGFR (epidermal growth factor receptor) monoclonal antibody treatment: in a recent paper [84], patients were excluded from anti-EGFR treatment based on hotspot sequencing for activating KRAS mutations and instead received chemotherapy, while the tumor-phenotyping, based on parallel reaction monitoring (PRM) mass spectrometry assay, indicated that patients might have benefitted from anti-EGFR therapy. The authors performed KRAS proteogenomic phenotyping, detecting more than 9,000 proteins characterized by considerable expression changes, including numerous ones involved in progression and resistance in CRC. Proteogenomic integration not only prioritized genomically inferred targets, such as copy number drivers and mutation-derived neoantigens, but also yielded novel findings.

Proteogenomics presents new avenues for biological discoveries and therapeutic development. As we have already remarked, right-sided colon cancer has a different prognosis compared to left-sided and rectal ones. A 2018 study [85] revealed significant differences between them: few unique molecular features of each tumor sub-types, not only at somatic but also at the proteomic level, were found. The individual role, played by each common tumor- initiating event (involving APC, KRAS, and TP53 genes), their order in tumor development, and selection of downstream somatic alterations were distinct in all three anatomical locations, discovering new significantly mutated genes at each tumor location. Some similarities were noted between left colon and rectal cancer. Proteogenomic characterization plus integrative and comparative genomic analysis provides a functional context to annotate genomic abnormalities with prognostic value.

\section{Acknowledgments}

We wish to thank Mrs. Claudia Cirillo (MA in English and Consultant for Strategic Affairs, Thomas Jefferson University, Philadelphia, PA, USA) for her English language editing of our text.

\section{Declaration of interest}

The authors have no relevant affiliations or financial involvement with any organization or entity with a financial interest in or financial conflict with the subject matter or materials discussed in the manuscript. This includes employment, consultancies, honoraria, stock ownership or options, expert testimony, grants or patents received or pending, or royalties.

\section{Reviewer disclosures}

Peer reviewers on this manuscript have no relevant financial or other relationships to disclose.

\section{References}

Papers of special note have been highlighted as either of interest $(\bullet)$ or of considerable interest $(\bullet)$ to readers.

1. Arnold M, Sierra MS, Laversanne $M$, et al. Global patterns and trends in colorectal cancer incidence and mortality. Gut. 2017;66:683-691.

2. De Greef K, Rolfo C, Russo A, et al. Multidisciplinary management of patients with liver metastasis from colorectal cancer. World J Gastroenterol. 2016;22:7215-7225.

3. Duan L, Yang W, Wang $X$, et al. Advances in prognostic markers for colorectal cancer. Expert Rev Mol Diagn. 2019;19:313-324.

-. This paper presented a very clear summary of different genetic, proteomic, epigenetic, immune and microbial biomarkers based on their own specific features.

4. Issa IA, Noureddine M. Colorectal cancer screening: an updated review of the available options. World J Gastroenterol. 2017;23:5086-5096.

5. Chauvin A, Boisvert F-M. Clinical proteomics in colorectal cancer, a promising tool for improving personalised medicine. Proteomes. 2018;6:49.

6. Frago R, Ramirez E, Millan M, et al. Current management of acute malignant large bowel obstruction: a systematic review. Am J Surg. 2014;207:127-138.

7. Ogino S, Giannakis M. Immunoscore for (colorectal) cancer precision medicine. Lancet. 2018;391:2084-2086.

8. Cuyle P-J, Prenen H. Current and future biomarkers in the treatment of colorectal cancer. Acta Clin Belg. 2017;72:103-115.

- This article focused on the possibility to use biomarkers to guide treatment choices in order to set patient-tailored therapies. 
9. Vasaikar S, Huang C, Wang X, et al. Proteogenomic analysis of human colon cancer reveals new therapeutic opportunities. Cell. 2019;177:1035-1049.

10. Das V, Kalita J, Pal M. Predictive and prognostic biomarkers in colorectal cancer: a systematic review of recent advances and challenges. Biomed Pharmacother. 2017;87:8-19.

11. Menter DG, Davis JS, Broom BM, et al. Back to the colorectal cancer consensus molecular subtype future. Curr Gastroenterol Rep. 2019;21:5.

12. Hanahan D, Weinberg RA. Hallmarks of cancer: the next generation. Cell. 2011;144:646-674.

13. Balacescu O, Sur D, Cainap C, et al. The impact of miRNA in colorectal cancer progression and its liver metastases. Int J Mol Sci. 2018;19:3711.

14. Gherman A, Balacescu L, Gheorghe-Cetean S, et al. Current and new predictors for treatment response in metastatic colorectal cancer. The role of circulating miRNAs as biomarkers. Int J Mol Sci. 2020;21:2089.

. This recent article highlighted the important role of miRNAs in the current clinical practice.

15. Brenner H, Kloor M, Pox CP. Colorectal cancer. Lancet Lond Engl. 2014;383:1490-1502.

16. Lynch HT, de la Chapelle A. Hereditary colorectal cancer. N Engl J Med. 2003;348:919-932.

17. Yang SY, Cho MS, Kim NK. Difference between right-sided and left-sided colorectal cancers: from embryology to molecular subtype. Expert Rev Anticancer Ther. 2018;18:351-358.

18. Natsume S, Yamaguchi T, Takao M, et al. Clinicopathological and molecular differences between right-sided and left-sided colorectal cancer in Japanese patients. Jpn J Clin Oncol. 2018;48:609-618.

19. The Lancet Oncology. Colorectal cancer: a disease of the young? Lancet Oncol. 2017;18:413.

20. Gupta N, Kupfer SS, Davis AM. Colorectal cancer screening. JAMA. 2019;321:2022-2023.

21. Nguyen MT, Weinberg DS. Biomarkers in colorectal cancer screening. J Natl Compr Canc Netw. 2016;14:1033-1040.

22. Weiser MR. AJCC 8th edition: colorectal cancer. Ann Surg Oncol. 2018;25:1454-1455.

23. Yan D, Yang X, Duan Y, et al. Comparison of laparoscopic complete mesocolic excision and traditional radical operation for colon cancer in the treatment of stage III colon cancer. J BUON Off J Balk Union Oncol. 2020;25:220-226.

24. Dekker E, Tanis PJ, Vleugels JLA, et al. Colorectal cancer. Lancet. 2019;394:1467-1480.

25. Rickard MJFX, Keshava A, Toh JWT. Three steps and a join: a simple guide to right- and left-sided medial to lateral laparoscopic colorectal surgery. Tech Coloproctol. 2017;21:673-677.

26. Ali SM, Pawlik TM, Rodriguez-Bigas MA, et al. Timing of surgical resection for curative colorectal cancer with liver metastasis. Ann Surg Oncol. 2018;25:32-37.

27. Ruan H, Leibowitz BJ, Zhang L, et al. Immunogenic cell death in colon cancer prevention and therapy. Mol Carcinog. 2020 Mar 25;59:783-793. [published ahead of print].

28. Yang $X$, Liu M, Li M, et al. Epigenetic modulations of noncoding RNA: a novel dimension of cancer biology. Mol Cancer. 2020;19:64.

29. Yu IS, Cheung WY. Metastatic colorectal cancer in the era of personalized medicine: a more tailored approach to systemic therapy. Can J Gastroenterol Hepatol. 2018;2018:9450754.

30. Surinova S, Choi M, Tao S, et al. Prediction of colorectal cancer diagnosis based on circulating plasma proteins. EMBO Mol Med. 2015;7:1166-1178.

31. Chen J, Sun H, Tang W, et al. DNA methylation biomarkers in stool for early screening of colorectal cancer. J Cancer. 2019;10:5264-5271.

32. Surinova S, Radová L, Choi M, et al. Non-invasive prognostic protein biomarker signatures associated with colorectal cancer. EMBO Mol Med. 2015;7:1153-1165.

33. De' Angelis GL, Bottarelli $L$, Azzoni $C$, et al. Microsatellite instability in colorectal cancer. Acta Bio Medica Atenei Parm. 2018;89:97-101.
34. Voorneveld PW, Jacobs RJ, Kodach LL, et al. A meta-analysis of SMAD4 immunohistochemistry as a prognostic marker in colorectal cancer. Transl Oncol. 2015;8:18-24.

35. Andreyev HJ, Norman AR, Cunningham $D$, et al. Kirsten ras mutations in patients with colorectal cancer: the «RASCAL \|» study. $\mathrm{Br}$ J Cancer. 2001;85:692-696.

36. Tong G, Xu W, Zhang G, et al. The role of tissue and serum carcinoembryonic antigen in stages I to III of colorectal cancer a retrospective cohort study. Cancer Med. 2018;7:5327-5338.

37. Okamura R, Hasegawa S, Hida K, et al. The role of periodic serum CA19-9 test in surveillance after colorectal cancer surgery. Int J Clin Oncol. 2017;22:96-101.

38. van der Waals LM, Laoukili J, Jongen JMJ, et al. Differential anti-tumour effects of MTH1 inhibitors in patient-derived 3D colorectal cancer cultures. Sci Rep. 2019;9:819.

39. Safaie Qamsari E, Safaei Ghaderi S, Zarei B, et al. The c-Met receptor: implication for targeted therapies in colorectal cancer. Tumour Biol $J$ Int Soc Oncodevelopmental Biol Med. 2017;39:1010428317699118.

40. Li X-L, Zhou J, Chen Z-R, et al. P53 mutations in colorectal cancer molecular pathogenesis and pharmacological reactivation. World J Gastroenterol. 2015;21:84-93.

41. Vedeld HM, Nesbakken A, Lothe RA, et al. Re-assessing ZNF331 as a DNA methylation biomarker for colorectal cancer. Clin Epigenetics. 2018;10:70.

42. Kuan T-C, Lin P-C, Yang S-H, et al. Impact of LINE-1 hypomethylation on the clinicopathological and molecular features of colorectal cancer patients. PloS One. 2018;13:e0197681.

43. Lech G, Słotwiński R, Słodkowski M, et al. Colorectal cancer tumour markers and biomarkers: recent therapeutic advances. World J Gastroenterol. 2016;22:1745-1755.

44. Henry NL, Hayes DF. Cancer biomarkers. Mol Oncol. 2012;6:140-146.

45. Ten Hoorn S, Trinh A, de Jong J, et al. Classification of colorectal cancer in molecular subtypes by immunohistochemistry. Methods Mol Biol. 2018;1765:179-191.

- This is a complete overview of CMSs different subtypes in CRC patients.

46. Linnekamp JF, van Hooff SR, Prasetyanti PR, et al. Consensus molecular subtypes of colorectal cancer are recapitulated in in vitro and in vivo models. Cell Death Differ. 2018;25:616-633.

47. Ioannidis JPA, Bossuyt PMM. Waste, leaks, and failures in the biomarker pipeline. Clin Chem. 2017;63:963-972.

48. Valle L, de Voer RM, Goldberg Y, et al. Update on genetic predisposition to colorectal cancer and polyposis. Mol Aspects Med. 2019;69:10-26.

49. Afkhami $E$, Heidari $M M$, Khatami $M$, et al. Detection of novel mitochondrial mutations in cytochrome $C$ oxidase subunit 1 (COX1) in patients with familial adenomatous polyposis (FAP). Clin Transl Oncol. 2020;22:908-918.

50. Sinicrope FA. Lynch syndrome-associated colorectal cancer. N Engl J Med. 2018;379:764-773.

51. Jiang Y-L, Zhao Z-Y, Li B-R, et al. The altered activity of P53 signaling pathway by STK11 gene mutations and its cancer phenotype in Peutz-Jeghers syndrome. BMC Med Genet. 2018;19:141.

52. Ma H, Brosens LAA, Offerhaus GJA, et al. Pathology and genetics of hereditary colorectal cancer. Pathology. 2018;50:49-59.

53. Maida $M$, Morreale $G$, Sinagra $E$, et al. Quality measures improving endoscopic screening of colorectal cancer: a review of the literature. Expert Rev Anticancer Ther. 2019;19:223-235.

54. Castro G, Azrak MF, Seeff LC, et al. Outpatient colonoscopy complications in the CDC's colorectal cancer screening demonstration program. Cancer. 2013;119:2849-2854.

55. Sun M, Liu J, Hu H, et al. A novel panel of stool-based DNA biomarkers for early screening of colorectal neoplasms in a Chinese population. J Cancer Res Clin Oncol. 2019;145:2423-2432.

56. Wang $\mathrm{W}, \mathrm{Xu} X$, Tian $\mathrm{B}$, et al. The diagnostic value of serum tumor markers CEA, CA19-9, CA125, CA15-3, and TPS in metastatic breast cancer. Clin Chim Acta. 2017:470:51-55. 
57. Gao Y, Wang J, Zhou Y, et al. Evaluation of serum CEA, CA19-9, CA72-4, CA125 and ferritin as diagnostic markers and factors of clinical parameters for colorectal cancer. Sci Rep. 2018;8:2732.

58. Bustamante-Lopez LA, Nahas SC, Nahas CSR, et al. Is there a difference between right versus left-sided colon cancers? Does side make any difference in long-term follow-up? Arq Bras Cir Dig. 2019;32:e1479.

59. Takahashi Y, Sugai T, Habano W, et al. Molecular differences in the microsatellite stable phenotype between left-sided and right-sided colorectal cancer. Int J Cancer. 2016;139:2493-2501.

60. Alves Martins BA, de Bulhões GF, Cavalcanti IN, et al. Biomarkers in colorectal cancer: the role of translational proteomics research. Front Oncol. 2019;9:1284.

61. Salem ME, Puccini A, Tie J. Redefining colorectal cancer by tumor biology. Am Soc Clin Oncol Educ Book. 2020;40:1-13.

62. Akkad J, Bochum S, Martens UM. Personalized treatment for colorectal cancer: novel developments and putative therapeutic strategies. Langenbecks Arch Surg. 2015;400:129-143.

63. Iorio J, Lastraioli E, Tofani L, et al. hERG1 and HIF-2a behave as biomarkers of positive response to bevacizumab in metastatic colorectal cancer patients. Transl Oncol. 2020;13:100740.

64. Ren D, Lin B, Zhang $X$, et al. Maintenance of cancer stemness by miR-196b-5p contributes to chemoresistance of colorectal cancer cells via activating STAT3 signaling pathway. Oncotarget. 2017;8:49807-49823.

65. Fujio A, Usuda M, Ozawa Y, et al. A case of gastrointestinal bleeding due to right hepatic artery pseudoaneurysm following total remnant pancreatectomy: a case report. Int J Surg Case Rep. 2017;41:434-437.

66. Qin Y, Li L, Wang F, et al. Knockdown of Mir-135b sensitizes colorectal cancer cells to oxaliplatin-induced apoptosis through increase of FOXO1. Cell Physiol Biochem. 2018;48:1628-1637.

67. Qian X, Yu J, Yin Y, et al. MicroRNA-143 inhibits tumor growth and angiogenesis and sensitizes chemosensitivity to oxaliplatin in colorectal cancers. Cell Cycle Georget Tex. 2013;12:1385-1394.

68. Wang K-Y, Ma J, Zhang F-X, et al. MicroRNA-378 inhibits cell growth and enhances L-OHPinduced apoptosis in human colorectal cancer. IUBMB Life. 2014;66:645-654.

69. Liu C, Eng C, Shen J, et al. Serum exosomal miR-4772-3p is a predictor of tumor recurrence in stage II and III colon cancer. Oncotarget. 2016;7:76250-76260.

70. Li LS, Morales JC, Veigl M, et al. DNA mismatch repair (MMR)-dependent 5-fluorouracil cytotoxicity and the potential for new therapeutic targets. Br J Pharmacol. 2009;158:679-692.

71. Caso R, Fabrizio A, Sosin M. Prolonged follow-up of colorectal cancer patients after 5 years: to follow or not to follow, that is the question (and how)! Ann Transl Med. 2020;8:164.
72. Boeckx N, Op de Beeck K, Beyens M, et al. Mutation and methylation analysis of circulating tumor DNA can be used for follow-up of metastatic colorectal cancer patients. Clin Colorectal Cancer. 2018;17:369-379.

73. Ramphal W, Boeding JRE, van Iwaarden $M$, et al. Serum carcinoembryonic antigen to predict recurrence in the follow-up of patients with colorectal cancer. Int J Biol Markers. 2019;34:60-68.

74. Barault L, Amatu A, Siravegna G, et al. Discovery of methylated circulating DNA biomarkers for comprehensive non-invasive monitoring of treatment response in metastatic colorectal cancer. Gut. 2018;67:1995-2005.

75. Zhang D, Zhao L, Zhou P, et al. Circulating tumor microemboli (CTM) and vimentin+ circulating tumor cells (CTCS) detected by a size-based platform predict worse prognosis in advanced colorectal cancer patients during chemotherapy. Cancer Cell Int. 2017;17:6.

76. Koncina E, Haan S, Rauh S, et al. Prognostic and predictive molecular biomarkers for colorectal cancer: updates and challenges. Cancers (Basel). 2020;12:319.

77. de Cuba EMV, Snaebjornsson P, Heideman DA, et al. Prognostic value of BRAF and KRAS mutation status in stage II and III microsatellite instable colon cancers. Int J Cancer. 2016;138:1139-1145.

78. Lee J-H, Ahn J, Park WS, et al. Colorectal cancer prognosis is not associated with BRAF and KRAS mutations - a STROBE compliant study. J Clin Med. 2019;8(1):pii: E111.

79. Zhao M, Mishra L, Deng C-X. The role of TGF- $\beta$ /SMAD4 signaling in cancer. Int J Biol Sci. 2018;14:111-123.

80. Vedeld HM, Merok M, Jeanmougin $M$, et al. CpG island methylator phenotype identifies high risk patients among microsatellite stable BRAF mutated colorectal cancers. Int J Cancer. 2017;141:967-976.

81. Jimenez CR, Knol JC, Meijer GA, et al. Proteomics of colorectal cancer: overview of discovery studies and identification of commonly identified cancer-associated proteins and candidate CRC serum markers. J Proteomics. 2010;73:1873-1895.

82. Chen X, Sun J, Wang X, et al. A meta-analysis of proteomic blood markers of colorectal cancer. Curr Med Chem. 2020 Apr 26 [published ahead of print]. doi:10.2174/0929867327666200427094054.

83. Ma YS, Huang T, Zhong XM, et al. Proteogenomic characterization and comprehensive integrative genomic analysis of human colorectal cancer liver metastasis. Mol Cancer. 2018;17:139.

84. Blank-Landeshammer B, Richard VR, Mitsa G, et al. Proteogenomics of colorectal cancer liver metastases: complementing precision oncology with phenotypic data. Cancers (Basel). 2019;11(12):pii: E1907.

85. Imperial R, Ahmed Z, Toor OM, et al. Comparative proteogenomic analysis of right-sided colon cancer, left-sided colon cancer and rectal cancer reveals distinct mutational profiles. Mol Cancer. 2018;17:177. 